2. improving the quality of life of the children and their families and carers.

Methods A literature search, with strict inclusion criteria, was done of the Cochrane Library, PubMed, and NICE guidelines, and articles were cross-referenced. The articles included were then critically appraised.

Results There are currently no evidence-based clinical guidelines to advise doctors, patients and their families appropriately about oral feeding versus gastrostomy feeding for children with cerebral palsy. Analysis of the articles included in this review consistently supported gastrostomy as being beneficial compared with oral feeding for most, though not all, of the children in these studies, and their families. Nutritional status, subcutaneous fat deposition, weight gain, limb growth, and the number of hospital admissions for chest infections improved in children with gastrostomy tube insertion, and this improvement was significant and clinically important. Moreover, the majority of carers reported that, after gastrostomy insertion, children felt better, were more sociable, had improved general health, and that family life was improved with feeding being made easier and quicker.

Conclusion Gastrostomy tube feeding remains an important alternative nutritional source for children with cerebral palsy. It has consistently been shown to be beneficial at improving not only weight gain and growth, but also the quality of life for both the child and their carers in the long-term. However, there has been individual variability with regard to carers' perceptions of gastrostomy feeding, and mothers can often initially express a feeling of culpability for their child's poor growth and the need for gastrostomy surgery as a failure on their part. Each case needs to be viewed in the context of the social and cultural belief systems of each child's family, and multi-disciplinary support is therefore needed in order to help families with the decision-making process on a case-by-case basis. Furthermore, in the absence of guidelines currently, clinicians must be aware of the current "best evidence" to inform individual choice on gastrostomy as an aid to feeding.

\section{G63(P) DISEASE PATTERN, BACTERIOLOGICAL PROFILE AND OUTCOME OF CHILDHOOD ACUTE BACTERIAL MENINGITIS}

A Bari, F Zeeshan, A Mushatq, M Nazar. Pediatric Medicine, Children's Hospital, Lahore, Pakistan

\subsection{6/archdischild-2015-308599.62}

Aim To determine the disease pattern, etiological agents and outcome of childhood acute bacterial meningitis

Study design Descriptive observational study.

Place and duration of study

The Children's Hospital and Institute of Child Health,

Lahore, from Jan, 2012 to Dec, 2012.

Statistical analysis SPSS 16.0 version

Results Out of 199 pateints 36\% were female and 64\% male. 68.4\% were between 2 months and 1 year

$26.6 \%$ were between 1.1 and 3 years

$10 \%$ were between 3.1 and 5 years

97\% presented with fever

$87 \%$ with poor feeding

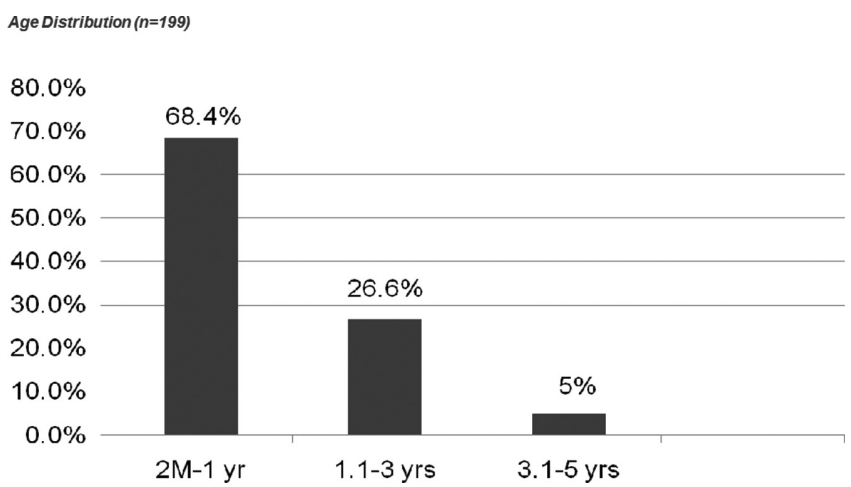

Abstract G63(P) Figure 1

Abstract G63(P) Figure 2 Microbiological diagnosis based on CSF cultures according to age 


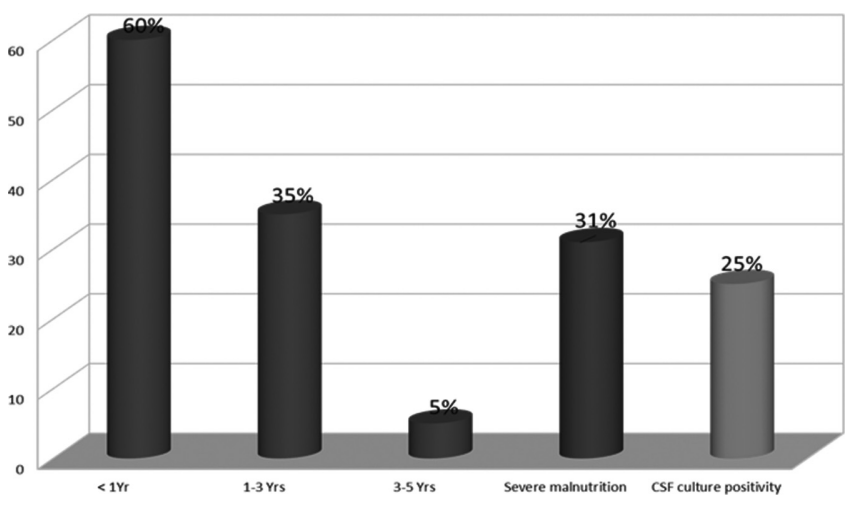

Abstract G63(P) Figure 3 Deaths in correlation with age, severe malnutrition and CSF culture positivity $n=20$

$25 \%$ with fits

$10 \%$ with crying

$23 \%$ with altered conscious level

$13 \%$ with signs of mniengeal irritation

Vaccination was incomplete in $7 \%$ unvaccinated $45 \%$ and vaccinated $48 \%$.

Complications $1 \%$ had hydrcephalus, $0.5 \%$ had subdural effusions, $4.5 \%$ had motor loss $10 \%$ have seizures maximum no of deaths less than 1 year. Malnutrition was associated with deaths in $31 \%$ and csf culture positivity in 25\% (Figures 1, 2, 3).

Conclusion Maxium no of deaths in males under 1 year, 33\% with malnutritiion, $25 \%$ with csf culture positivitity coagulase negative staph aureus and streptoccoccus pneumnia were two most commoon pathogens in culture.

Mortality under one year was maximun and associated with streptococcus pneumonia.

\section{G64(P) FOCAL SEIZURE, HEMIPARASIS, HEMIPLAGIA AND NMDA RECEPTOR ANTIBODY ENCEPHALITIS - ATYPICAL PRESENTATION}

D Onyekwere, G Margabanthu. Paediatrics, Kettering General Hospital, Kettering, UK

\subsection{6/archdischild-2015-308599.63}

Aim We present an atypical case of NMDAR Ab Encephalitis (N-methyl-D-Aspartate Receptor Antibody Encephalitis) with recurrent right sided focal seizures, right hemiparesis and hemiplegia. The youngest case in UK to be described without significant psychiatric component.

Methods 4 years 10 month old girl born in UK to Zimbabwean Parents. She had been well with not relevant family history. She presented with right sided focal seizure and reduced movement. She had 2 episodes of generalised tonic convulsions involving the right side associated with loss of consciousness which lasted for $2 \mathrm{~min}$ and then $10 \mathrm{~min} ; 5 \mathrm{~min}$ apart. The seizures was managed with diazepam. No preceding trauma, fever, neck pain, headache or vomiting. Her right sided weakness which improved within $2 \mathrm{~h}$.

She was then re admitted 4 days later with right focal seizure with persisting right sided weakness, dysarthria and right sided facial palsy. She had amnesia without any new psychotic features

Baseline blood and CSF Investigation were normal. Lyme serology, ASOT, Lupus screen, all viral PCR and throat swab were negative. ECG, Echo and EEG was normal. CSF amino acid, ammonia, Lyme titre, oligoclonal bands, mitochondrial
DNA, acylcarnitine profile was normal. MRI showed possible left MCA infarct with a picture of encephalitis with oedema.

She was treated with antibiotics, lorazepam, Aspirin and Omeprazole. Repeat MRI ruled out a stroke so Aspirin was stopped and started on IV methylprednisolone.

Repeated MRI brain suggested an inflammatory process with evidence of maturing damage suggestive of neurometabolic or neuroinflammatory disorder. Importantly the CSF and blood NMDAR antibody returned positive with rising titres of blood NMDAR ab.

Results NMDA Receptor Antibody Encephalitis was diagnosed and treated with Prednisolone $30 \mathrm{mg}$ OD for 8/52, advised avoiding live vaccines and IVIG $1 \mathrm{~g} / \mathrm{kg}$ for $2 / 7$. MMF $200 \mathrm{mg}$ BD planned for 18-24 months- dose to be adjusted based on response. Monthly NMDAR ab blood and CSF have been positive for 10 months after diagnosis. She is on Annual surveillance for tumours with MRI or USS. She continues to have physiotherapy and Neuropsychology input.

Conclusion Atypical focal seizures may need further evaluation and NMDAR ab considered with atypical presentations resulting in better outcome.

\section{G65(P) NEUROPSYCHIATRIC PRESENTATION OF ANTIBODY NEGATIVE LIMBIC ENCEPHALITIS}

K Moodley, A Menounou, A Kirby, A Durso, D Krishnakumar. Paediatrics and Child Health, Colchester General Hospital, Colchester, UK

\subsection{6/archdischild-2015-308599.64}

Autoimmune encephalitis is an increasingly recognised cause for a combination of symptoms of seizures, disturbance of memory, behaviour and cognition. Other manifestations include acute onset movement disorders, unexplained encephalopathy and refractory status epilepticus. N-methyl aspartate (NMDA) encephalitis and voltage gated potassium channel mediated (VGKC) limbic encephalitis are the two commonest causes of autoimmune encephalitis. These children initially present to general paediatricians therefore it is vital to consider this in the differential diagnosis of infective encephalitis as prompt recognition, investigation and immune therapy determines longterm outcome. Early recognition and treatment can potentially halt temporal lobe atrophy and improve outcome.

We report a 14 year old girl who presented with symptomatic, intermittent memory loss, behaviour disturbance, fatigue and cognitive change with limbic encephalitis. Initial investigations revealed negative PCR for HSV, EBV. Brain imaging detected mesial right temporal high signal area in FLAIR sequences. EEG showed epileptic inter-ictal focal abnormality over right frontal mesial temporal region. NMDA, VGKC, Anti GAD, Hu, Ma, CV2, CRMP5 and thyroid antibodies were negative. Screening for tumour with baseline abdominal ultrasound and CXR were negative. Baseline WISC testing prior to starting medications revealed her immediate memory for verbal information was in the low average range (verbal immediate index score:82,12th percentile) and her time delayed recall verbal information was in the impaired range (verbal delayed index score:72, 3rd percentile). She was treated with iv methylprednisolone followed by tapering dose of oral prednisolone. Repeat WISC assessment is due in 6 months.

Limbic encephalitis should be considered in children with prominent neuropsychiatric manifestation of encephalitis. Her future MRI reports will determine the 3 month and 6 month 